\title{
Standard Bundles Simplify Standards-based Quantification in NIST DTSA-II
}

\author{
Nicholas W. M. Ritchie ${ }^{1}$, Michael J. Mengason ${ }^{1}$ and Dale E. Newbury ${ }^{1}$ \\ ${ }^{1}$ National Institute of Standards and Technology, Gaithersburg, MD 20899-8371
}

One of the design goals for the X-ray microanalysis software NIST DTSA-II (National Institute of Standards and Technology DTSA-II, a pseudo-acronym) was to make standards-based quantification based on $k$-ratios as simple as possible. (DTSA-II is freely available and can be downloaded from http://www.cstl.nist.gov/div837/837.02/epq/dtsa2/index.html.) Standards-based quantifi-cation has the potential to be the most accurate way to make electron excited X-ray microanalysis measurements[1, 2, 3]. In the standards-based k-ratio protocol, the k-ratio is defined as the ratio of the intensity of characteristic X-ray line over the intensity of the same characteristic X-ray line measured on the standard, $k=I_{\text {unk }} / I_{\text {std }}$.

Standards-based quantification can be quite simple if pure element or simple, non-interfering compounds are used. Interferences occur when the characteristic X-ray line from one element is sufficiently close in energy to a characteristic X-ray line from another element. To resolve both element's characteristic lines, there needs to be a stretch of largely unobstructed continuum background between the peaks. Accurate measurement of the characteristic X-ray intensity is only possible when there is an accurate measurement of the continuum in the range of energies surrounding the peak.

While it is possible to find pure element or simple compound standards which satisfy the noninterference requirement, these standards usually don't produce the most accurate measurements. Accuracy in mi-croanalysis is a function both of the statistical precision of the standard and unknown spectra but also the accuracy of the matrix correction used to compensate for differences in electron and x-ray transport properties between the standards and unknown materials. The most accurate measurement can usually be performed using standard materials which are similar in composition to the unknown. These similar stan-dards (alternatively matrix matched standards) often have interferences between two or more elements that make fitting them directly to an unknown unfeasible.

There are different strategies to resolve interferences. DTSA-II's primary strategy is to use distinct spectra called reference spectra to resolve the interferences. Consider two elements $Z_{1}$ and $Z_{2}$ with a mutual interference. If pure element spectra can be collected for $Z_{1}$ and $Z_{2}$, the pure element spectra can be used as intermediaries between the standard spectra and the unknown spectra. Fit the pure element spectra for $Z_{1}$ and $Z_{2}$ to the standard to produce k-ratios $k_{Z_{1}, \text { std }}$ and $k_{Z_{2} \text { std }}$. Fit the pure element spectra to the unknown to produce k-ratio $k_{Z_{1}, \text { unk }}$ and $k_{Z_{2}, \text { unk }}$. The k-ratios for the unknown relative to the standard are then $k_{Z_{1}}=k_{Z_{1} \text {,unk }} / k_{Z_{1} \text {,std }}$ and $k_{Z_{2}} \stackrel{=}{=} k_{Z_{2} \text {,unk }} / k_{Z_{2} \text { stdd }}$. Furthermore, the reference spectra don't need to be spectra from pure elements. Almost any material with un-obstructed characteristic lines can be used as a reference. Often this means binary compounds, sometimes even compounds that are too unstable under the electron beam to be suitable for standards.

An alternative strategy is to use a theoretical model of the continuum and characteristic X-ray line shapes in place of the reference spectrum. This strategy can be challenging to implement because the detectors have artifacts and the relative weights of the characteristic lines are insufficiently well-known. The non-linearity of the detector calibration can shift the perceived characteristic X-ray energy from true energy by a channel or two at certain energies. Shifts deleteriously effect the transfer efficiency of modeled reference spectra and bias the resulting k-ratios. Furthermore, it is necessary to allow the weights-of-lines to be varied around the best known values. To handle this complexity, DTSA-II uses a constrained, gradient, non-linear fit algorithm to fit the detector resolution, calibration, peak intensities, continuum model and weights-of-lines. This fit has many adjustable parameters and works well in most situations but can fail in certain pernicious edge cases. Particularly problematic is fitting two elements 
with characteristic line energies that are very similar. Performing the fit in multiple steps can resolve some problems by first fitting global parameters like detector performance and continuum model before relaxing constraints to fit additional parameters like relative weights-of-lines.

DTSA-II has long permitted the use of reference spectra to resolve interferences present in the standards [4]. However, quantifying spectra using complex standards requiring many references can be tedious and complex. It was often frustrating to learn late into the quantification process that an additional reference spectrum was required. As a team that includes both users and user/developers, frustrations from the users were quickly communicated to the developers and the users made suggestions on how they'd like to see the software improved. This close collaboration lead to an effective way to resolve the reference problem and to streamline quantification.

The idea is simple. The interferences in the standard determine the required references so consolidate these spectra (standard and references) into a file called a standard bundle. The best time to create a standard bundle is when the standard spectra are collected and before quantifying unknown spectra. The standard bundle provides a tool to sum multiple individual spectra into a single standard. Combining multiple spectra collected from various points on the standard is an excellent way to ensure the consistency of standard spectra. Ideally, the variance between point spectra should be similar to the variance from count statistics alone. The tool checks whether all the necessary information in the spectra is both present and consistent. The tool determines when references are required and checks whether spectra are suitable for use as reference. The tool lists the required references and allows you to either 1) specify a spectrum to use as reference, or 2) fit a modeled spectrum to the standard and extract a reference from the modeled spectrum. The first case is usually preferable to the second because it is likely to produce more accurate results. However, the second method is provided for cases in which a suitable reference can not be found.

The validated information including standard and reference spectra and an XML header that describes the contents is written into a standard bundle file with an extension ".zstd". The quantification tool has been enhanced to accept standard bundles in place of individual standard and reference spectra. This simple enhancement greatly simplifies and expedites high quality standards-based quantification of unknown spectra.

\section{References:}

[1] V. D. Scott, G. Love, and S. J. B. Reed. Quantitative Electron-Probe Microanalysis. Ellis Horwood, second edition, 1995.

[2] J. Goldstein et al,. Scanning Electron Microscopy and X-Ray Microanalysis. Kluwer Academic/ Plenum Publishers, New York, 3rd. edition, 2003.

[3] S. J. B. Reed. Electron Microprobe Analysis and Scanning Electron Microscopy in Geology: Cambridge University Press, 2nd. edition, July 2005.

[4] Dale E. Newbury and Nicholas W. M. Ritchie. Journal of Materials Science 50(2) (2015), p. 493. 